\section{Oral contraceptives, ABO blood types and thromboembolism}

An interesting article in $C M A J$ by Sode and colleagues ${ }^{1}$ reported that in groups of patients with increased susceptibility to thromboembolic disease because of factor V Leiden R 5062 or prothrombin mutation G 20210A, the highest incidence of disease occurred in patients with $\mathrm{A}, \mathrm{B}$ or $\mathrm{AB}$ blood types. In previous studies, ${ }^{2-4}$ we reported that among 348 women treated with various oral contraceptive agents and observed for 5877 months, thromboembolic complications occurred only in patients with preexisting blood coagulation deficiencies, but all patients had $\mathrm{A}$ or $\mathrm{AB}$ blood types.

The coagulation deficiencies we observed produced no clinical problems until oral contraceptives were initiated. Abnormalities included increased levels of factors V, VII, VIII, II (prothrombin) or its mutations or decreased levels of plasminogen or combinations of these pathologies. The question is whether patients should be tested for factors of the blood coagulation and fibrinolysin systems as well as blood groups before starting oral contraceptive therapy. Although the incidence of thromboembolism in these patients is relatively low, and the tests are expensive, the potential of serious complications in otherwise healthy women may warrant testing. Positive findings may justify consideration of other methods of pregnancy prevention, or of the use of oral contraceptives with the lowest estrogen concentrations, which have the lowest risk of thromboembolic complications.

\section{Julian L. Ambrus MD PhD}

Professor of Internal Medicine, SUNY at Buffalo, Buffalo General Hospital,

Buffalo, NY

\section{References}

1. Sode BF, Allin KH, Dahl M, et al. Risk of venous thromboembolism and myocardial infarction associated with V Leiden and prothrombin mutations and blood type. CMAJ 2013;185:E229-37.

2. Ambrus CM, Lillie MA, Akhter S. Studies on oral contraceptives and thromboembolism. J Med 2006; 37:117-28.

3. Ambrus MS, Mink IB. ABO blood types and fac- tors of the blood coagulation and fibrinolysin systems in women. J Med 1970;1:236-41.

4. Lillie MA, Mink IB. Progestational agents and blood coagulation VI relationship of abo blood types to changes induced by oral contraceptives. Am J Obstet Gynecol 1974;119:406-10.

CMAJ 2013. DOI:10.1503/cmaj.113-2148

\section{Guidelines and public consultations}

In response to the CMAJ commentary by Chatterjee and colleagues, ${ }^{1}$ we at BC Guidelines wish to inform the authors that we have used their suggested method of public consultation for our guidelines for the past 10 years.

BCGuidelines.ca is overseen by the Guidelines and Protocols Advisory Committee (GPAC), a joint committee of the BC Medical Association and the British Columbia Ministry of Health. New and revised guidelines are subject to an external peer review to ensure guidelines are clearly written, appropriate, practical, and free from serious oversights or errors.

Each guideline is written by a working group of general practitioners, relevant specialists, a pharmacist and a research officer. The working group reviews current evidence and drafts the guideline, which is reviewed internally by GPAC and then sent for external review. The external review consists of mailing the guideline and accompanying questionnaire to a random sample of general practitioners (between 400 and 800 individuals), relevant specialties (10\%-20\% sample per specialty) that include nurse practitioners, other allied health professionals and stakeholders. The stakeholders are key contacts in the areas of pharmacy, laboratory procedures, health authorities, medical services plan billing, public health and health professional colleges and associations.

The questionnaire consists of approximately 10 questions that address clarity, applicability, utilization and overall assessment. Space is given for openended comments. The feedback from the external review is discussed by the working group, and any necessary changes are made. Before a guideline can be finalized by GPAC and the Medical Services Commission of British Columbia, the feedback received from the external review must be reconciled.

Further information about BC Guidelines can be found at BCGuidelines.ca or hlth.guidelines@gov.bc.ca.

\section{Bakul I. Dalal MD, Stephanie Power MA}

BC Medical Association, Guidelines and Protocols Advisory Committee CoChair (Dalal); and British Columbia Ministry of Health, Guidelines and Protocols Advisory Committee Cochair (Power).

\section{Reference}

1. Chatterjee A, Bhattacharyya O, Persaud N. How can Canadian guideline recommendations be tested? CMAJ 2013;185:465-7.

CMAJ 2013. DOI:10.1503/cmaj.113-2145

\section{Interpreting positive urine cultures}

On behalf of emergency physicians at Hamilton Health Sciences, we are compelled to respond to an article ${ }^{1}$ that appeared in the practice section of CMAJ. In a survey of our colleagues, 29 out of 30 respondents believed that the patient in the article by Vaisman and colleagues ${ }^{1}$ had a urinary tract infection. Only 1 respondent thought that the positive culture represented asymptomatic bacteriuria.

Because of the high mortality rate due to bacterial infection among older adults, and because systemic inflammatory response syndrome and septic shock were the most immediate and life-threatening possible diagnoses, most physicians would have likely treated the patient with antibiotics upon presentation.

Of the studies cited in the CMAJ article, ${ }^{1}$ one was a qualitative (i.e., taperecorded interview) study of nurses and doctors describing their diagnostic and prescribing practices concerning bacteriuria within a nursing home setting, ${ }^{2}$ another was a self-report study from 1987 of 72 elderly participants' (59 women and 13 men) symptoms and urine culture results, in which there was no control for cognitive ability to describe symptoms. ${ }^{3}$ 
Vaisman and colleagues ${ }^{1}$ justify their decision to deny the patient antibiotic therapy based on prevalence-biased diagnostic measures, low probability of disease in a different (i.e., otherwise healthy) population and by retroductive inference.

Because the patient presented in a shock state with evidence of urosepsis, she required immediate treatment for sepsis, including antibiotics, and, in our opinion, treating her otherwise was unacceptable.

\section{Margaret J. Ackerman MD MEd,} Andrew Worster MD, Daren Lin MD Emergency physician (Ackerman, Worster, Lin), Hamilton Health Sciences, Hamilton, Ont.

\section{References}

1. Vaisman A, Gold WL, Leis JA. A 78-year-old woman with lethargy and a positive urine culture. CMAJ 2013; 185:679-80

2. Walker S, McGeer A, Simor AE, et al. Why are antibiotics prescribed for asymptomatic bacteriuria in institutionalized elderly people? A qualitative study of physicians' and nurses' perceptions. CMAJ 2000;163:273-7.

3. Nicolle LE, Mayhew WJ, Bryan L. Prospective randomized comparison of therapy and no therapy for asymptomatic bacteriuria in institutionalized elderly women. Am J Med 1987;83:27-33.

CMAJ 2013. DOI:10.1503/cmaj.113-2146

\section{The authors respond}

We thank Ackerman and colleagues ${ }^{1}$ for their discussion related to our arti$\mathrm{cle}^{2}$ and for taking the time to poll their colleagues on how they would have managed the patient in the clinical scenario. Interpretation of positive urine culture results has long been the subject of passionate debate in the literature. ${ }^{3}$

Because we believe that a change in clinical practice regarding urine culture test ordering and management of positive results is needed, we are encouraged that our article has stimulated a healthy discussion on the issue.

We agree with the use of early empiric antibiotic therapy in reducing mortality due to sepsis; however, in the scenario presented, the patient did not meet criteria for sepsis. ${ }^{4}$ Furthermore, an alternate explanation existed for the patient's hypotension and tachycardia, namely hypovolemia secondary to gastrointestinal fluid losses. Although the patient had a fever 3 days before presentation, it was self-limited and there was an alternate explanation - her diarrheal illness in the setting of an institutional outbreak. This also showed that she could mount a febrile response in the event of an infection. Therefore, we disagree with Ackerman and colleagues" conclusion that the patient had systemic inflammatory response syndrome (SIRS) and septic shock and required early antibiotic therapy.

If the patient had met the case definition for SIRS and sepsis, an additional point to highlight would be that older adults with sepsis without urinary symptoms should not be assumed to have a urinary infection based on findings of urinalysis or culture results, because this may lead to early diagnostic closure and a failure to investigate and treat for other causes of infection.

Bacteriuria is present in up to $50 \%$ of elderly women in long-term care facilities; and $90 \%$ of those patients have pyuria. ${ }^{5}$ Therefore, these abnormalities should not constitute a urinary tract infection diagnosis in a patient who can reliably report the presence or absence of urinary symptoms, as in the case presented. ${ }^{2}$

Our case highlights the importance of appreciating clinical context when both ordering urine cultures and interpreting results. In the absence of urinary symptoms and the presence of an alternate diagnosis, the positive culture in the patient likely represented asymptomatic bacteriuria, rather than a urinary tract infection. Robust clinical literature, including many randomized controlled trials, show the lack of benefit of treating asymptomatic bacteriuria with antimicrobial therapy across multiple patient populations. ${ }^{6-8}$ Furthermore, antimicrobial therapy for asymptomatic bacteriuria has been associated with harm. One trial showed an increased risk of symptomatic urinary tract infection in those who were treated for asymptomatic bacteriuria. ${ }^{9}$ The practice is also associated with adverse drug reactions and Clostridium difficile infection. ${ }^{10}$

Alon Vaisman MD, Wayne L. Gold MD, Jerome A. Leis MD

Department of Medicine (Vaisman, Gold,

Leis), University of Toronto; and the

Division of Infectious Diseases (Gold, Leis), University Health Network, Toronto, Ont.

\section{References}

1. Ackerman MJ, Worster A, Lin D. Interpreting positive urine cultures [letter]. CMAJ 2013;185:1526-7.

2. Vaisman A, Gold WL, Leis JA. A 78-year-old woman with lethargy and a positive urine culture. CMAJ 2013; 185:679-80.

3. Miller J. To treat or not to treat: managing bacteriuria in elderly people. CMAJ 2001;164:619-20.

4. Dellinger RP, Levy MM, Rhodes A, et al. Surviving Sepsis Campaign: international guidelines for management of severe sepsis and septic shock. 2012. Intensive Care Med 2013;39:165-228.

5. Nicolle LE, Bradley S, Colgan R. Infectious Diseases Society of America Guidelines for the Diagnosis and Treatment of Asymptomatic Bacteriuria in Adults. Clin Infect Dis 2005; 40:643-54.

6. Nicolle LE, Bjornson J, Harding GK, et al. Bacteriuria in elderly institutionalized men. $N$ Engl J Med 1983;309:1420-5.

7. Boscia JA, Kobasa WD, Knight RA, et al. Therapy v. no therapy for bacteriuria in elderly ambulatory nonhospitalized women. JAMA 1987;257:1067-71.

8. Abrutyn E, Mossey J, Berlin JA, et al. Does asymptomatic bacteriuria predict mortality and does antimicrobial treatment reduce mortality in elderly ambulatory women? Ann Intern Med 1994; 120:827-33.

9. Cai T, Mazzoli S, Mondaini N, et al. The role of asymptomatic bacteriuria in young women with recurrent urinary tract infections: to treat or not to treat? Clin Infect Dis 2012;55:771-7.

10. Rotjanapan P, Dosa D, Thomas KS. Potentially inappropriate treatment of urinary tract infections in two Rhode Island nursing homes. Arch Intern Med 2011;171:438-43

CMAJ 2013. DOI:10.1503/cmaj.113-2147

\section{Letters to the editor}

In submitting a letter, you automatically consent to have it appear online and/or in print. All letters accepted for print will be edited by $C M A J$ for space and style. Most references and multiple authors' names, full affiliations and competing interests will appear online only. (The full version of any letter accepted for print will be posted at cmaj.ca.) 